\title{
A MESH SIZE CONTROL PARAMETER FOR A FINITE ELEMENT APPROXIMATION OF THE HELMHOLTZ EQUATION
}

\author{
J. E. Sebold ${ }^{1}$, L. A. Lacerda ${ }^{2}$, J.A.M. Carrer ${ }^{3}$ \\ ${ }^{1}$ Programa de Pós-Graduação em Métodos Numéricos em Engenharia, Universidade Federal \\ do Paraná (jeanseboldl@gmail.com)
}

${ }^{2}$ Programa de Pós-Graduação em Métodos Numéricos em Engenharia, Universidade Federal do Paraná (alkimin@ lactec.org.br)
${ }^{3}$ Programa de Pós-Graduação em Métodos Numéricos em Engenharia, Universidade Federal do Paraná (carrer@ufpr.br)

\begin{abstract}
Resumo. Este artigo trata de um Método de Elementos Finitos usando funções de base hierárquicas de Legendre para a aproximação da solução da equação Helmholtz unidimensional. As condições de contorno acústicas são fracamente representadas, e é definido um problema auxiliar com condições de contorno homogêneas facilitando o caminho para a aproximação. Esta abordagem auxiliar permite a formulação de um método de solução geral. Elementos finitos de segunda ordem são usados juntamente com um parâmetro de discretização com base no número de onda fixado e da tolerância de erro requerida. Uma fórmula explícita é definida para o controle do tamanho do parâmetro da malha baseado na aproximação de Padé. A análise paramétrica é conduzida para validar a abordagem de elementos finitos e o parâmetro de controle de malha. O controle da dispersão nos resultados mostra que a formulação numérica é robusta e pode ser estendida para ordens superiores na análise de elementos finitos.
\end{abstract}

Keywords: Elementos Finitos, Base Hierárquica, Aproximação de Padé.

\section{INTRODUÇÃO}

Neste trabalho faremos uso do Método dos Elementos Finitos com a ideia de aproximar soluções para a equação de Helmholtz que descreve o fenômeno acústico em uma dimensão. Para tanto, utilizaremos algumas propostas vistas anteriormente, por exemplo, Harari e Thompson [3], estudaram a estrutura acústica envolvendo modelos de radiação e espalhamento, e Ainsworth [1], que analisou a dispersão discreta para aproximação por elementos finitos em números de onda relativamente altos. 
O Método dos Elementos Finitos usando funções de base hierárquicas tem a propriedade de que a base não precisa de ser reconstituída, quando o grau do polinômio é aumentado. Haverá, apenas, um acréscimo de funções às já existentes. Esta propriedade é muito útil quando se utiliza a versão-p do Método dos Elementos Finitos. As funções de forma para uma base hierárquica são definidas como integrais de polinômios de Legendre, que possuem diversas propriedades interessantes, como por exemplo, a ortogonalidade e outras que veremos a seguir.

\section{BASE HIERÁRQUICA DE LEGENDRE}

Seja $\mathcal{S}^{p} \subset H^{1}(\Omega)$ um subespaço de elementos finitos do espaço dos polinômios contínuos por partes de grau $p$ denotado por $\mathcal{P}^{p}$.

$$
\mathcal{S}^{p}=\left\{\phi \mid \phi \in C_{0}^{1}(\Omega), \phi \in \mathcal{P}^{p}\left(\Omega_{e}\right)\right\},
$$

onde $C_{0}^{1}(\Omega)$ é o espaço de todas as funções que são uma vez continuamente diferenciáveis, as quais juntamente com suas derivadas, tem suporte compacto em $\Omega$, que é um aberto limitado de $\mathbb{R}$ e $\Omega_{e}=[-1,1]$ é o elemento de referência.

Seja $\left\{L_{k}\right\}_{k=1}^{p}$ o conjunto dos polinômios de Legendre, os quais são dados pela fórmula de Rodrigues para $0 \leq k \leq p$

$$
L_{k}(\xi)=\frac{1}{2^{k} k !} \frac{d^{(k)}}{d \xi^{k}}\left[\left(\xi^{2}-1\right)^{k}\right], \quad \xi \in[-1,1]
$$

além disso, vamos definir o conjunto $\left\{N_{k}\right\}_{k=1}^{p+1}$ como

$$
\left\{\begin{array}{l}
N_{k}(\xi)=\frac{1}{2}\left(1+\xi_{k} \xi\right), \quad k=1,2, \quad \text { onde } \quad \xi_{1}=1 \quad \text { e } \quad \xi_{2}=-1 . \\
N_{k}(\xi)=\frac{1}{\left\|L_{k-2}\right\|} \int_{-1}^{\xi} L_{k-2}(t) d t, \quad k=3, \ldots, p+1, \quad\left\|L_{k-2}\right\|^{2}=\frac{2}{2 k-3}
\end{array}\right.
$$

Note que $N_{k}( \pm 1)=0$ para $k>2$. Como resultado desta propriedade, as variáveis $\phi_{1}=\phi_{h}(-1)$ e $\phi_{2}=\phi_{h}(1)$ definirão as variáveis nodais discretas, enquanto que $\phi_{k}$, com $k \geq 3$, formarão um conjunto de variáveis internas.

As funções definidas por (2), formam o que chamamos de base hierárquica de Legendre $[3,8]$. As funções de forma, ou de base, nodais são dadas pela variação $k=1,2$, enquanto que para $k=3, \ldots, p+1$, temos as funções de base internas.

As derivadas destas funções hierárquicas formam uma base ortogonal com a seguinte propriedade:

$$
\int_{-1}^{1} \frac{d N_{a}}{d \xi} \frac{d N_{b}}{d \xi} d \xi=\delta_{a b} \quad a, b=3, \ldots, p+1,
$$

onde $\delta_{a b}$ é o delta de Kronecker. 


\subsection{Experimentos Numéricos}

Em uma dimensão, a equação de onda acústica harmônica no tempo pode ser escrita na seguinte forma [4]

$$
\phi^{\prime \prime}+\kappa^{2} \phi+Q \delta\left(x, x_{Q}\right)=0 \quad \forall x \in \Omega=(0,1),
$$

onde $\phi(x)$ é a pressão acústica complexa, $\kappa$ é o número de onda e $Q \delta\left(x, x_{Q}\right)$ é uma fonte sonora constante localizada em $x_{Q}$. A equação (4) é conhecida como a Equação de Helmholtz não-homogênea. Considerando a fonte sonora desprezível, queremos aproximar $\phi \in H^{1}(\Omega)$, tal que

$$
\left(\phi^{\prime}, w^{\prime}\right)+\kappa^{2}(\phi, w)=0
$$

para todo $w \in H_{0}^{1}(\Omega)$ sujeito às condições de contorno(C.C.)

$$
\text { C.C. }\left\{\begin{array}{l}
\text { Pressão : } \quad \phi=\tilde{\phi} \quad \forall x \in \Gamma \\
\text { Velocidade }: \quad \frac{d \phi}{d x}=\frac{d \tilde{\phi}}{d x}=i \kappa \rho v(x) \quad \forall x \in \Gamma
\end{array}\right.
$$

onde $\Gamma$ é o contorno de $\Omega, \rho$ é a densidade de massa $\left(\rho=1,29 \mathrm{~kg} / \mathrm{m}^{3}\right.$ para o ar em $0^{\circ} \mathrm{C}$ e 1-atm), $v(x)$ é a velocidade e a quantidade $\tilde{\phi}$ é uma função complexa dada.

Se escolhermos $\kappa=10 \pi$ e $v(x)=e^{i \kappa x}$, então uma solução exata para o problema (5) com condições de contorno (6) pode ser dada por $\tilde{\phi}(x)=\rho e^{i \kappa x}$.

Vamos apresentar agora uma forma de resolver o problema (5)-(6). A ideia é definir, a partir de $\tilde{\phi}(x)$ dado, um novo problema com condições de contorno homogêneas. Aproximaremos o novo problema pelo método de elementos finitos utilizando as funções de base hierárquicas de Legendre de segunda ordem, logo após, usaremos a aproximação obtida para recuperar a solução do problema (5)-(6). Para tanto, vamos definir $\alpha(x)=1+\frac{\operatorname{sen}\left(\pi x^{2}\right)(x-1)}{n}$, onde $n$ é o número de elementos na malha, em seguida definimos também $\phi_{0}=\alpha(x) \tilde{\phi}$ e, por fim, $\bar{\phi}=\tilde{\phi}-\phi_{0}$. Deste modo, temos que na fronteira de $\Omega, \bar{\phi}(0)=\bar{\phi}(1)=\frac{d \bar{\phi}(0)}{d x}=\frac{d \bar{\phi}(1)}{d x}=0$. Portanto, $\bar{\phi}$ satisfaz o seguinte problema variacional: Encontrar $\bar{\phi} \in H_{0}^{1}(\Omega)$ tal que

$$
\left(\bar{\phi}^{\prime}, w^{\prime}\right)+\kappa^{2}(\bar{\phi}, w)=(f, w)
$$

onde $f(x)=\left(\frac{d^{2} \alpha(x)}{d x^{2}}\right) \tilde{\phi}+2\left(\frac{d \alpha(x)}{d x} \frac{d \tilde{\phi}}{d x}\right)$, para todo $w \in H_{0}^{1}(\Omega)$ sujeito às condições de contorno(C.C.)

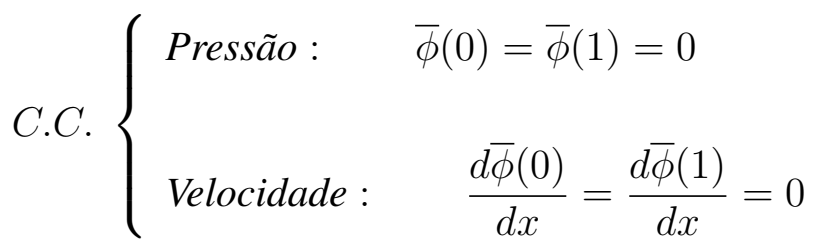

Ao discretizar o domínio $\Omega=(0,1)$, devemos calcular a aproximação por elementos 
finitos de segunda ordem $\bar{\phi}_{h 2} \in \mathcal{S}^{2} \cap H_{0}^{1}(\Omega)$ tal que

$$
\left(\bar{\phi}_{h 2}^{\prime}, w^{\prime}\right)+\kappa^{2}\left(\bar{\phi}_{h 2}, w\right)=(f, w)
$$

para todo $w \in \mathcal{S}^{2} \cap H_{0}^{1}(\Omega)$. Aproximando o problema (7)-(8) com $\bar{\phi}_{h 2}$, recuperaremos a solução aproximada para o problema (5)-(6) fazendo $\tilde{\phi}_{h 2}=\bar{\phi}_{h 2}+\phi_{0}$.

A Figura 1 - (a), (b), (c), (d), (e) e (f) mostra as aproximações por elementos finitos de segunda ordem utilizando funções de base hierárquicas de Legendre de acordo com os níveis de refinamento.

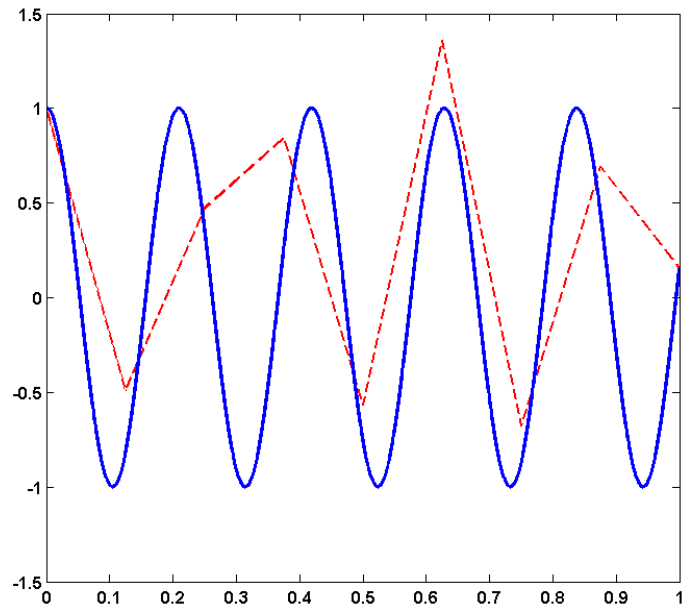

(a)

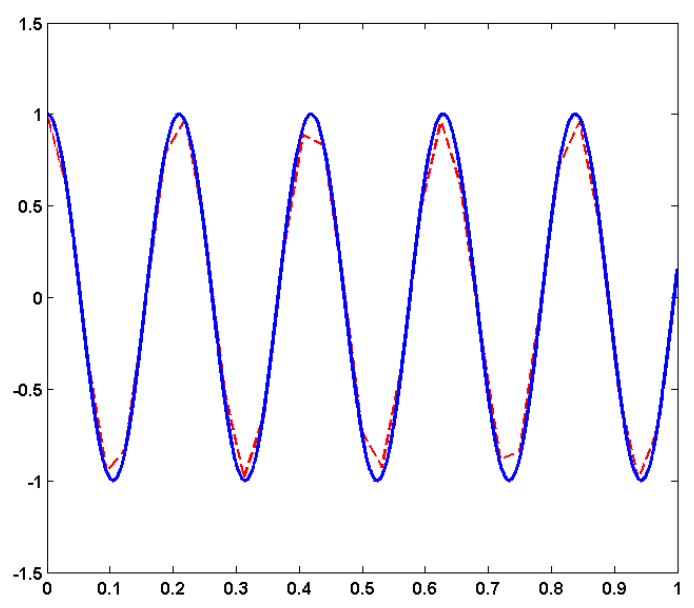

(c)

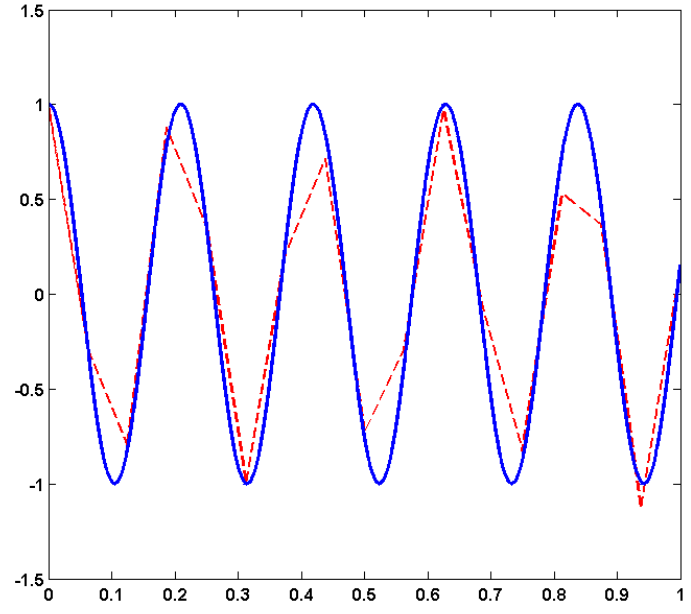

(b)

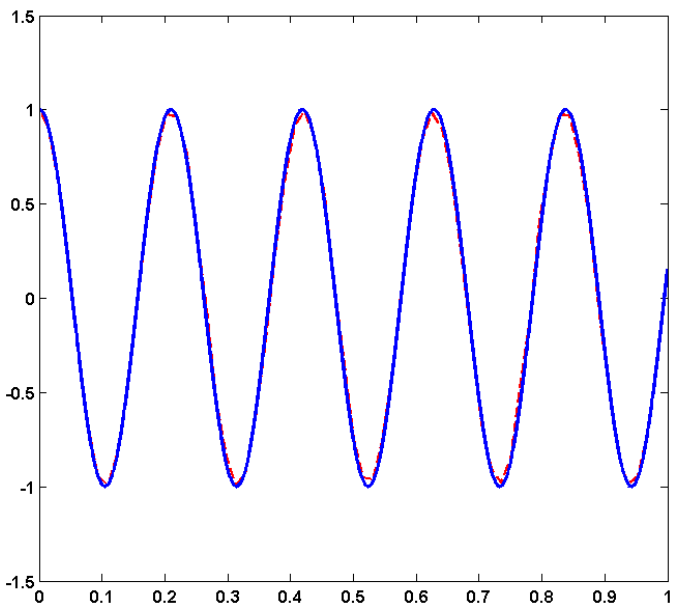

(d) 


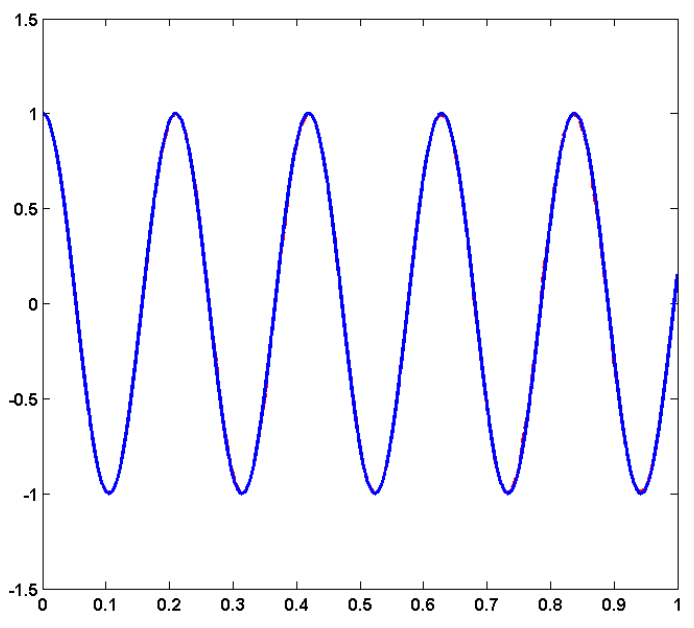

(e)

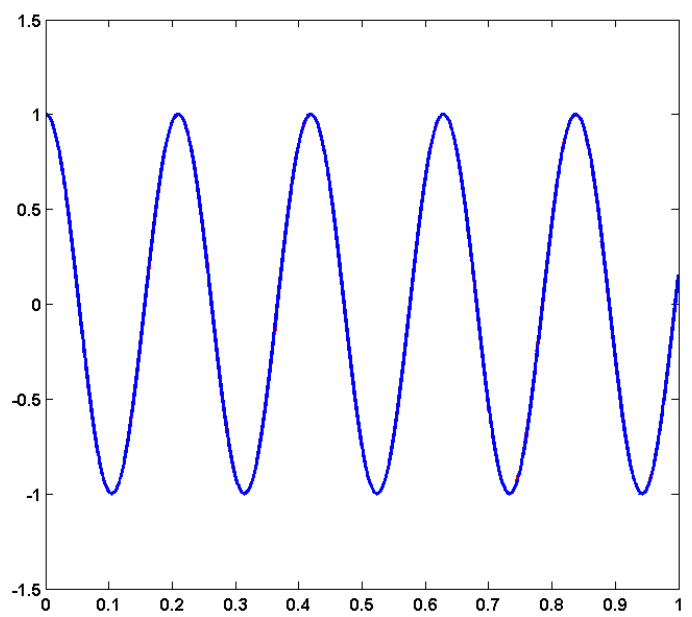

(f)

Figura 1. Os níveis de refinamento são: (a) $h=1 / 4,(b) h=1 / 8,(c) h=1 / 16,(d) h=1 / 32,(e) h=1 / 64$, (f) $h=1 / 128$. A solução exata corresponde a linha contínua, enquanto que a solução aproximada corresponde com a linha tracejada.

\section{RELAÇÃO DE DISPERSÃO DISCRETA}

$\mathrm{Na}$ seção anterior podemos observar na Figura 1 - (a), (b) e (c), uma diferença de fase entre a solução exata e aproximada, tal diferença diminui a medida que o refinamento da malha aumenta Figura 1 - (d), (e) e (f). Com o objetivo de analisar o erro através da diferença entre a velocidade de fase do problema contínuo e a velocidade de fase numérica da aproximação, faremos nesta seção um estudo da relação de dispersão.

Seja $\Omega \subset \mathbb{R}$ um domínio com contorno $\Gamma$. Em uma dimensão, a equação de onda acústica homogênea [6] pode ser escrita como

$$
\frac{\partial^{2} u}{\partial x^{2}}-\frac{1}{c^{2}} \frac{\partial^{2} u}{\partial t^{2}}=0
$$

Para ondas harmônicas no tempo, as soluções para (10) podem ser escritas como

$$
u(x, t)=\tilde{u}(x) e^{-i \omega t}
$$

onde $\tilde{u}(x)$ é a pressão acústica no domínio da frequência, $\omega$ é a frequência circular, $c$ é a velocidade do som e $i^{2}=-1$. Substituindo (11) em (10), tem-se

$$
\tilde{u}^{\prime \prime}(x)+\kappa^{2} \tilde{u}(x)=0, \quad \operatorname{com} \kappa=\omega / c \text { e } \forall x \in \Omega,
$$

onde $\kappa$ é o número de onda que está relacionado com $\omega$ pela relação de dispersão [1]

$$
\omega=|\kappa|
$$

A equação (12) é conhecida como Equação Escalar de Helmholtz Homogênea em uma di- 
mensão.

Suponha agora que uma malha uniforme de tamanho $h>0$ está colocada sobre a reta real com nós localizados em $h \mathbb{Z}$, onde $\mathbb{Z}$ é o conjunto dos inteiros. Denotemos $V_{h}$ o conjunto funções lineares contínuas por partes relativas a malha. Em analogia ao problema contínuo (10), buscaremos soluções da forma

$$
u_{h}(x, t)=\tilde{u}_{h}(x) e^{-i \omega t}
$$

de modo que $\tilde{u}_{h} \in V_{h}$ deva satisfazer

$$
B\left(\tilde{u}_{h}, v_{h}\right)=0 \quad \forall v_{h} \in V_{h}
$$

onde

$$
B\left(\tilde{u}_{h}, v_{h}\right)=\left(\tilde{u}_{h}^{\prime}, v_{h}^{\prime}\right)-\kappa^{2}\left(\tilde{u}_{h}, v_{h}\right)
$$

e $(\cdot, \cdot)$ denota o produto interno $L_{2}$ sobre $\mathbb{R}$.

A translação invariante da malha sugere que busquemos ondas de Bloch[5] como soluções, na forma

$$
\tilde{u}_{h}(x)=\sum_{n \in \mathbb{Z}} e^{i n k h} \theta_{n}(x)
$$

onde $\theta_{n}$ é uma função linear contínua por partes definida como

$$
\theta_{n}(s)=\left\{\begin{array}{ccc}
1-\left(n-\frac{s}{h}\right) & \text { se } & s \in(n h-h, n h] \\
1+\left(n-\frac{s}{h}\right) & \text { se } & s \in(n h, n h+h) \\
0 & \text { caso contrário } &
\end{array}\right.
$$

com a propriedade

$$
\theta_{n}(x+m h)=\theta_{n-m}(x), \quad x \in \Omega \text { e } m \in \mathbb{Z}
$$

a qual implica que $\tilde{u}_{h}$ possui a propriedade característica da onda de Bloch, ou seja, para cada $n \in \mathbb{Z}$, tem-se

$$
\tilde{u}_{h}(x+n h)=e^{i \kappa n h} \tilde{u}_{h}(x), \quad x \in \Omega
$$

A função $\tilde{u}_{h} \in V_{h}$ pode ser interpretada como a versão discreta da exponencial complexa $\tilde{u}(s)=e^{i \kappa s}$. Motivado pelo fato que $\tilde{u}(s)$ é a solução da equação escalar de Helmholtz em uma dimensão [2],

$$
\tilde{u}^{\prime \prime}+\omega^{2} \tilde{u}=0
$$

aqui adotamos $\kappa^{2}=\omega^{2}$, devido a relação de dispersão (13). Utilizando (16) podemos escrever 
o seguinte problema variacional: Encontrar $\tilde{u}_{h} \in V_{h}$ tal que

$$
\int_{\mathbb{R}} \tilde{u}_{h}^{\prime}(s) v_{h}^{\prime}(s) d s=\omega_{h}(\kappa)^{2} \int_{\mathbb{R}} \tilde{u}_{h}(s) v_{h}(s) d s \quad \forall v_{h} \in V_{h}
$$

onde $\omega_{h}(\kappa)^{2}$ pode ser calculado definindo $v_{h}=\theta_{m}$, com $m \in \mathbb{Z}$, na sequência substituindo (17) em (22) e observando que $\theta_{n}(s) \theta_{m}(s) \neq 0$ somente quando $s \in(m h-h ; m h+h)$ (o mesmo valendo para $\theta_{n}^{\prime}(s) \theta_{m}^{\prime}(s)$ ). Deste modo, encontramos

$$
\int_{(m-1) h}^{(m+1) h} \sum_{n=(m-1) h}^{(m+1) h} e^{i h \xi n} \theta_{n}^{\prime}(s) \theta_{m}^{\prime}(s) d s=\omega_{h}(\xi)^{2} \int_{(m-1) h}^{(m+1) h} \sum_{n=(m-1) h}^{(m+1) h} e^{i h \xi n} \theta_{n}(s) \theta_{m}(s) d s
$$

expandindo os somatórios e resolvendo os integrais, encontramos

$$
\omega_{h}(\kappa)^{2}=\frac{6}{h^{2}}\left(\frac{1-\cos (h \kappa)}{2+\cos (h \kappa)}\right)
$$

e se $h \kappa \ll 1$, podemos expandir (24) como série de Maclaurin, e assim

$$
\omega_{h}(\kappa)^{2}=\kappa^{2}\left(1+\frac{(h \kappa)^{2}}{12}+\ldots\right)
$$

logo, se $h \rightarrow 0$, tem-se no limite que

$$
\omega_{h}(\kappa)=\kappa \Longrightarrow \omega^{2}=\omega_{h}^{2}(\kappa)
$$

A expressão (26) corresponde a relação de dispersão discreta para a equação escalar de Helmholtz em uma dimensão.

Logo, para usar (24), gostaríamos que fosse possível expressar $\omega_{h}(\kappa)$ em termos de $\kappa$. Uma forma mais prática, para facilitar esta exibição, seria encontrar uma definição implícita para $\omega_{h}(\kappa)$ em termos de $\cos (h \kappa)$. Por exemplo, no caso de elementos de primeira $\operatorname{ordem}(p=$ 1), a relação (24) pode ser reescrita na forma

$$
\cos (h \kappa)=\frac{6-2\left(h \omega_{h}\right)^{2}}{6+\left(h \omega_{h}\right)^{2}}
$$

Esta expressão é generalizada para arbitrárias ordens de $p$ no seguinte teorema, que é demonstrado em [1].

Teorema 3.1 Sejam $\left[2 N_{e}+2 / 2 N_{e}\right]_{\kappa \tan (\kappa)}$ e $\left[2 N_{o} / 2 N_{o}-2\right]_{\kappa \cot (\kappa)}$ a notação para a aproximação de Padé de $\kappa$ tan $(\kappa)$ e $\kappa \cot (\kappa)$, respectivamente, onde $N_{e}=\lfloor p / 2\rfloor$ e $N_{o}=$ $\lfloor(p+1) / 2\rfloor$. Desta forma, $\omega_{h p}$ satisfaz $\cos (h \kappa) \approx R_{p}\left(h \omega_{h p}\right)$, onde $R_{p}$ é a função racional

$$
R_{p}(2 \kappa)=\frac{\left[2 N_{o} / 2 N_{o}-2\right]_{\kappa \cot (\kappa)}-\left[2 N_{e}+2 / 2 N_{e}\right]_{\kappa \tan (\kappa)}}{\left[2 N_{o} / 2 N_{o}-2\right]_{\kappa \cot (\kappa)}+\left[2 N_{e}+2 / 2 N_{e}\right]_{\kappa \tan (\kappa)}}
$$

A Figura 2, abaixo, mostra a aproximação $\cos (h \kappa) \approx R_{p}\left(h \omega_{h p}\right), \operatorname{com} p=1,2,3,4$. 

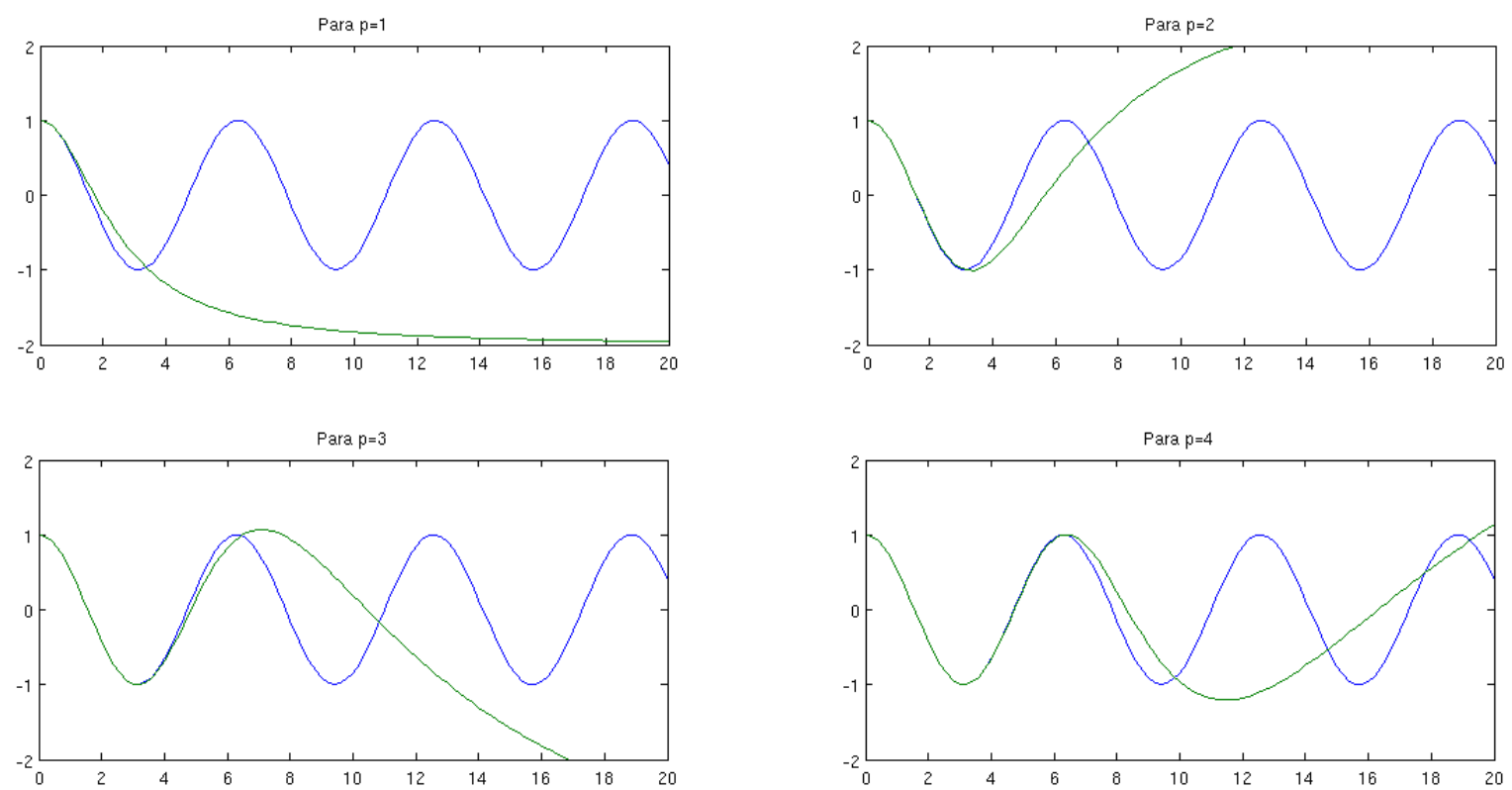

Figura 2. Curvas para $\cos (h \kappa)$ comparadas com as curvas produzidas pela função racional $R_{p}\left(h \omega_{h p}\right)$ dada em (3.1) para o método de ordem $p=1,2,3,4$.

Do teorema acima podemos construir a seguinte tabela para valores de $p=1,2,3,4$ :

\begin{tabular}{lc}
\hline$p$ & $\cos (h \kappa) \approx R_{p}\left(h \omega_{h p}\right)$ \\
\hline 1 & $\frac{6-2\left(h \omega_{h 1}\right)^{2}}{6+\left(h \omega_{h 1}\right)^{2}}$ \\
& $\frac{3\left(h \omega_{h 2}\right)^{4}-104\left(h \omega_{h 2}\right)^{2}+240}{\left(h \omega_{h 2}\right)^{4}+16\left(h \omega_{h 2}\right)^{2}+240}$ \\
3 & $\frac{-4\left(h \omega_{h 3}\right)^{6}+540\left(h \omega_{h 3}\right)^{4}-11520\left(h \omega_{h 3}\right)^{2}+25200}{\left(h \omega_{h 3}\right)^{6}+30\left(h \omega_{h 3}\right)^{4}+1080\left(h \omega_{h 3}\right)^{2}+25200}$ \\
4 & $\frac{5\left(h \omega_{h 4}\right)^{8}-1800\left(h \omega_{h 4}\right)^{6}+134064\left(h \omega_{h 4}\right)^{4}-2378880\left(h \omega_{h 4}\right)^{2}+5080320}{\left(h \omega_{h 4}\right)^{8}+48\left(h \omega_{h 4}\right)^{6}+3024\left(h \omega_{h 4}\right)^{4}+161280\left(h \omega_{h 4}\right)^{2}+5080320}$
\end{tabular}

TABELA 1: Casos especiais da aproximação racional $R_{p}\left(h \omega_{h p}\right)$ para o $\cos (h \kappa)$ de acordo com a ordem $p$ do método.

\section{SELEÇÃO DO PARÂMETRO DA MALHA}

Vamos utilizar a teoria desenvolvida nas duas seções anteriores para estabelecer um critério de seleção do parâmetro da malha $(h=1 / n)$ para elementos de segunda ordem [7].

A equação (26) descreve a relação de dispersão discreta $\omega^{2}=\omega_{h}^{2}(\kappa)$. Definindo agora a velocidade de fase numérica como sendo

$$
C=\frac{\omega_{h}(\kappa)}{\kappa},
$$


podemos considerar o caso com $p=2$ na Tabela 1 , onde

$$
\cos (h \kappa)=\frac{3\left(h \omega_{h 2}\right)^{4}-104\left(h \omega_{h 2}\right)^{2}+240}{\left(h \omega_{h 2}\right)^{4}+16\left(h \omega_{h 2}\right)^{2}+240}
$$

e na sequência escrever a velocidade de fase numérica $C$ em função de $h \kappa$, obtendo

$$
C=\frac{1}{h \kappa}\left[\frac{16 \cos (h \kappa)+104+\sqrt{(16 \cos (h \kappa)+104)^{2}-960(\cos (h \kappa)-3)(\cos (h \kappa)-1)}}{6-2(\cos (h \kappa))}\right]^{1 / 2}
$$

Por outro lado, pela relação de dispersão (13) do problema contínuo, temos que a velocidade de fase exata é dada por $c=1$. A figura 3(a) mostra a velocidade de fase exata e numérica. A figura 3(b) é uma ampliação da figura 3(a) que permite estimar, por exemplo, o maior valor possível do parâmetro $h$ de modo que o erro de fase estimado seja inferior a $0,01 \%$, bastando observar o ponto em que as curva de velocidade atinge o valor 1,0001 (ou seja, $h \kappa \approx 0,62$ ).

Na Figura 1, gráficos (e) e (f), temos um refinamento 1/64 e 1/128, respectivamente. Note que, se $\kappa=10 \pi$, então $h \approx \frac{0,62}{10 \pi} \approx 0,01973 \approx \frac{1}{51}$, ou seja, $1 / 64<0,01973$, assim como $1 / 128<0,01973$. Deste modo, podemos observar que para $n \geq 51$ a diferença de fase, no experimento numérico feito na seção 1 , é menor que $0,01 \%$. Deste modo, os gráficos (a), (b), (c) e (e), na Figura 1, apontam um erro superior a esta estimativa.

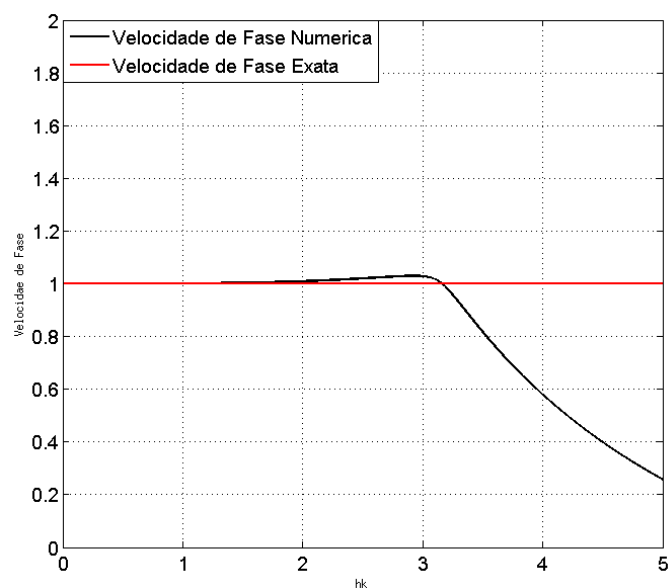

(a)

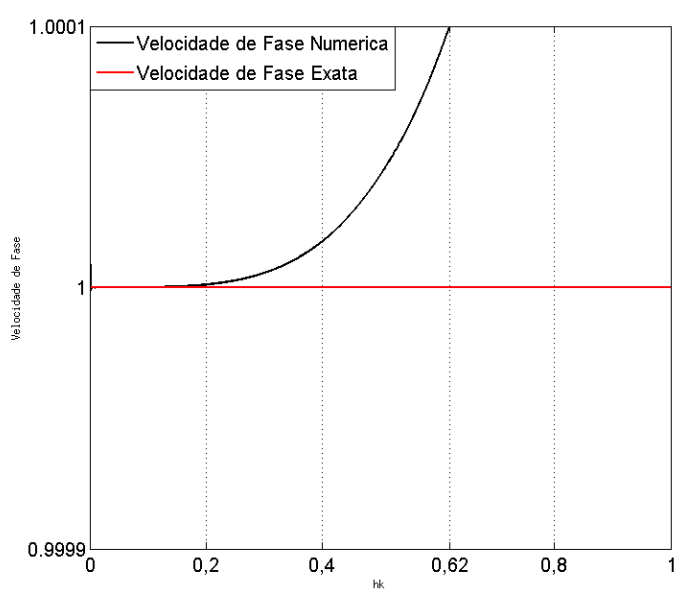

(b)

Figura 3. Velocidade de Fase dos Elementos de Segunda ordem.

\section{CONCLUSÕES}

Após descrever as funções de base hierárquicas de Legendre, realizamos alguns experimentos numéricos em um típico problema de ondas acústicas, no qual verificamos alguns interessantes resultados na aproximação. Tais resultados revelaram diferenças na fase da onda da solução aproximada, quando utilizamos condições de contorno não-homogêneas. 
Na sequência, vimos que a relação de dispersão discreta, para elementos de primeira ordem, foi descrita em termos da relação de dispersão discreta para a aproximação da equação escalar de Helmholtz em uma dimensão.

Por fim, trabalhamos na busca do mais adequado parâmetro da malha, fornecendo informações quantitativas sobre o seu nível de refinamento. Deste modo, podemos proporcionar uma forma de controlar os efeitos dispersivos na aproximação por elementos finitos.

Uma proposta para trabalhos futuros seria investigar o comportamento da aproximação por Elementos de Finitos utilizando funções de base hierárquicas de Fourier e funções de base de Lagrange com a quadratura de Lobatto, com o intuito de comparação com o método realizado neste trabalho.

\section{Referências}

[1] Ainsworth, M. Discrete dispersion for hp-version finite element approximation at high wavenumber. SIAM J. Numer. Analysis vol. 42, pp. 553-575, 2003. 1, 3, 3

[2] Ainsworth, M. Dispersive properties of high - order Nédélec/edge element approximation of the time - harmonic Maxwell equations. Philosophical transactions of the The Royal Society of London. vol. 362, pp. 471-491, 2004. 3

[3] Harari, I. and Thompson, L.L. Recent Developments in Finite Element Methods for Structural Acoustic. Archives of Computational Methods in Engineering. vol. 3, pp. 131311, 1996. 1,2

[4] Liu, Y. Fast Multipole Boundary Element Method. Cambridge University Press, New York, 2009. 2.1

[5] Odeh, F. and Keller, J.B. Partial differential equation. with periodic coeffcients and Bloch waves in crystals J. Math. Phys. vol. 5, pp. 1499-1504, 1964. 3

[6] Oliveira, S.P. and Seriani, G. Optimal blended spectral-element operators for acoustic wave modeling. Geophysics. vol. 72, no 5, pp. 95-106, 2007. 3

[7] Sebold, J. E. e Oliveira, S.P.(Orientador) Métodos de Elementos Finitos de Nédélec para as Equações de Maxwell Harmônicas no Tempo. Dissertação de Mestrado - Programa de Pós-Graduação em Matemática Aplicada - UFPR, Curitiba, 2011. 4

[8] Thompson, L. and Pinsky, P. Complex wavenumber Fourier analysis of the p-version finite element method. Computat. Mech. vol. 13, pp. 255-275, 1994. 2 